\title{
Saúde bucal na população indígena Xavánte de Pimentel Barbosa, Mato Grosso, Brasil
}

\author{
Oral health among the Xavánte Indians \\ in Pimentel Barbosa, Mato Grosso, Brazil
}

\begin{abstract}
Rui Arantes 1
Ricardo Ventura Santos 1,2

Carlos E. A. Coimbra Jr. 1
\end{abstract}

\footnotetext{
1 Escola Nacional de Saúde Pública, Fundação Oswaldo Cruz, Rua Leopoldo Bulhões 1480, Rio de Janeiro, RJ 21041-210, Brasil. ruiarantes@apcd.org.br 2 Departamento de Antropologia, Museu Nacional, Universidade Federal do Rio de Janeiro. Quinta da Boa Vista s/no. Rio de Janeiro, $R J$ 20940-040, Brasil. santos@ensp.fiocruz.br carlos_coimbrajr@gbl.com.br
}

\begin{abstract}
This study presents the results of an oral health epidemiological survey conducted in 1997, based on WHO criteria, in the Xavánte indigenous community of Pimentel Barbosa (or Etéñitépa), Mato Grosso State, Central Brazil. The study included 228 individuals (85\% of the population) over two years of age. In about half the sample, the DMF index was less than 2, and in the 12-14-year age bracket it was 3.7. The low frequency of fillings in permanent and deciduous teeth suggests limited access to dental care services. Despite the number of sextants with bleeding and tartar, no cases of severe periodontal disease were detected (CPITN). In the community, comparison of the results of this survey with two previous surveys (1962 and 1991) showed a deterioration in oral health conditions over time and alterations in the occlusal pattern (increase in Angle class II and III). Dietary changes due to environmental and socioeconomic alterations resulting from interaction with the surrounding society, along with the lack of preventive programs, are among the causes of this deterioration in oral health among the Xavante.
\end{abstract}

Key words Public Health Dentistry; Oral health; Xavánte; South American Indians

Resumo Em 1997, foi realizado um levantamento epidemiológico em saúde bucal na comunidade indígena Xavánte de Etéñitépa (ou Pimentel Barbosa), Mato Grosso, Brasil. Foram seguidos os critérios preconizados pela OMS. Aproximadamente metade da amostra $(N=228)$ apresentou CPOD inferior a 2. Em crianças de 12 a 14 anos, observou-se um CPOD de 3,7. O índice CEO foi mais elevado aos seis anos (5,6). A freqüência de dentes obturados é bastante reduzida, apontando para o pouco acesso a serviços odontológicos. As má-oclusões (classificação de Angle) apresentam baixa ocorrência, assim como a doença periodontal em estado avançado (avaliada pelo ICNTP). A comparação dos resultados do inquérito realizado em 1997 com levantamentos conduzidos em 1962 e 1991 revelou uma tendência de deterioração das condições de saúde bucal. Argumenta-se que mudanças na dieta (notadamente consumo de açúcar e de outros produtos industrializados), relacionadas a alterações sócio-econômicas e ambientais advindas da interação com a sociedade nacional envolvente, aliadas à falta de programas preventivos, estão entre as principais causas da deterioração nas condições de saúde bucal dos Xavánte.

Palavras-chave Odontologia em Saúde Pública; Saúde bucal; Xavánte; Índios Sul-Americanos 


\section{Introdução}

As condições de saúde bucal em nível individual e coletivo são avaliadas, principalmente, pela análise da ocorrência da cárie e de doenças periodontais. Por ser mais freqüente, a cárie tem sido intensamente estudada, do ponto de vista etiológico e epidemiológico, com o objetivo de melhor conhecer determinantes, prevalência e distribuição, assim como estabelecer medidas preventivas (Pinto, 1990; WHO, 1972, 1992). No Brasil, as informações epidemiológicas disponíveis sobre ocorrência e distribuição da cárie são ainda muito limitadas, no que diz respeito tanto à cobertura geográfica, como a tendências temporais. Usualmente, envolvem amostras urbanas, sobretudo de escolares (Dini et al., 1999; Freysleben et al., 2000; MS, 1988, 1996; Narvai et al., 2000; Normando \& Araújo, 1990; Pinto, 1992; Viegas \& Viegas, 1988).

Diversos autores têm apontado para a necessidade de pesquisas que contemplem grupos étnicos específicos, minorias e migrantes, dentre outros, a fim de contribuir para o melhor conhecimento acerca da multicausalidade da cárie e dos diferentes padrões assumidos por essa doença no mundo, bem como para levantar informações relevantes para o planejamento de serviços de saúde em contextos específicos (Pilot, 1988; Walker, 1987; Winter, 1990). No Brasil, por exemplo, é muito pouco conhecida a epidemiologia da saúde bucal entre os povos indígenas, o que reflete um quadro mais geral de desconhecimento sobre as condições de saúde dessas populações (Coimbra Jr. \& Santos, 2000). Estudos sobre a saúde bucal de povos indígenas têm sido esporádicos, restringindo-se quase sempre a trabalhos transversais, em amostras pequenas, e, em geral, enfatizando comunidades ainda relativamente semi-isoladas (Ayres \& Salzano, 1972; Detogni, 1994; Donnelly, 1977; Hirata et al., 1977; Niswander, 1967; Pereira \& Evans, 1975; Tricerri, 1985; Tumang \& Piedade, 1968). Desse quadro, resulta uma grande escassez de informações qualitativas e quantitativas sobre o estado de saúde bucal das populações indígenas no Brasil, apesar das intensas mudanças sócio-econômicas e ambientais pelas quais vêm passando esses povos, incluindo subsistência e dieta, que são aspectos reconhecidamente propiciadores de alterações no perfil de saúde bucal (Santos \& Coimbra Jr., 1994).

Este trabalho apresenta um levantamento das condições de saúde bucal de uma comunidade indígena Xavánte localizada em Mato Grosso, no Brasil Central. O contato permanen- te dos Xavánte com a sociedade nacional brasileira ocorreu em meados do século XX e, desde então, foram intensas as mudanças nas mais diversas esferas da vida cotidiana. A partir dos anos 60, os Xavánte que atualmente vivem na Terra Indígena (T. I.) Pimentel Barbosa foram investigados por diferentes grupos de pesquisadores, do ponto de vista antropológico (Maybury-Lewis, 1967), biomédico, inclusive odontológico, (Neel et al., 1964; Pose, 1993), ecológico (Flowers 1983; Santos et al., 1997) e nutricional (Flowers, 1983; Gugelmin, 1995). Tais estudos oferecem subsídios que possibilitam compreender os padrões de mudanças experimentados pelos Xavánte, permitindo, também, contextualizar os possíveis fatores sócio-econômicos e ambientais relacionados às condições de saúde bucal.

\section{População e Métodos}

A presente pesquisa foi desenvolvida na aldeia Xavánte Pimentel Barbosa (também conhecida como Etéñitépa), localizada na Terra Indígena (TI) Pimentel Barbosa (13o 20' S, 51o 40’ W), no leste de Mato Grosso. Este grupo está em contato permanente com a sociedade nacional brasileira desde a década de 40 (Flowers, 1983; Maybury-Lewis, 1967; Silva, 1992). Os Xavánte baseavam sua dieta na coleta de frutos e raízes silvestres, caça e horticultura, em especial, a do milho (Flowers, 1983; Giaccaria \& Heide, 1972; Maybury-Lewis, 1967). Atualmente, em virtude de uma série de modificações sócio-econômicas e ambientais resultantes do contato, a base da dieta passou a incluir, em larga medida, o arroz, cultivado em roças familiares e comunitárias (Flowers, 1983; Gugelmin, 1995; Santos et al., 1997). Ainda que os alimentos tradicionais continuem a ocupar importante espaço na dieta dos Xavánte de Etéñitépa, o consumo de alimentos industrializados vem aumentando de modo significativo nas últimas décadas (Gugelmin, 1995; Santos et al., 1997). No que se refere às práticas de higienização bucal preconizadas pela odontologia, os Xavánte não praticam, de forma generalizada, a escovação dentária. A água consumida pela população não é artificialmente fluoretada, originando-se de cursos d'água e de um poço.

O trabalho de campo, realizado de julho a setembro de 1997, envolveu o exame odontológico domiciliar de 228 pessoas $-47 \%$ do sexo masculino e $53 \%$ do sexo feminino -, constituindo cerca de $85 \%$ do total da população com idade superior a dois anos. Foram visitados os 23 domicílios da aldeia e examinados os pre- 
sentes. Ao analisar a composição etária da amostra, verificou-se que se aproxima daquela da população total. Assim, 62,2\% da amostra abrange idade inferior a 20 anos, ao passo que $42,5 \%$, inferior a 12 anos. Apenas $11,9 \%$ dos indivíduos têm idade superior a 40 anos.

O exame clínico, executado por um único investigador (Arantes), foi feito sob luz natural, utilizando-se espelho plano e sonda exploradora. O modelo das fichas, a definição da lesão de cárie (somente lesões cavitadas) e as demais variáveis observadas no exame clínico seguiram os critérios preconizados pela Organização Mundial da Saúde (WHO, 1987). As variáveis consideradas foram as seguintes: condições dentárias pelo índice CPOD, necessidade de tratamento, fluorose e condições periodontais, através do índice periodontal (ICNTP = índice comunitário de necessidades de tratamento periodontal), má oclusão, relação de molares (classificação de Angle), além do uso e necessidade de próteses.

Após ter sido conduzido o inquérito relacionado às condições de saúde bucal, efetuouse atendimento clínico a todos os indivíduos que manifestaram interesse. Este consistiu basicamente em remoção dos focos infecciosos e dos dentes irrecuperáveis, restauração dos dentes recuperáveis, curativos, pulpotomias e remoção de cálculo.

O banco de dados foi constituído mediante a utilização do programa DBase III (AshtonTate, 1985) e foi analisado através do programa Epi Info, versão 6.0 (WHO, 1994). Neste último, foram realizados o agrupamento da amostra por faixa etária e sexo, os cálculos dos índices CPOD e "ceo", com suas respectivas médias e desvios-padrão, e a distribuição das freqüências absolutas e relativas das variáveis estudadas.

\section{Resultados}

No total de 228 indivíduos examinados, observou-se a seguinte distribuição dos valores do CPOD: 0 (37,3\%), 1-2 (12,2\%), 3-4 (11,5\%), 5-7 (9,5\%), 8-10 (6,1\%), 11-15 (9,8\%), 16-20 (6,6\%) e $>21(7,0 \%)$. Portanto, cerca da metade da amostra apresentou CPOD inferior a 2. Para a faixa etária de 5 a 12 anos, a freqüência de indivíduos sem dentes cariados, perdidos ou obturados $(C P O D=0)$ foi de $67,5 \%$ (Tabela 1). Nessa mesma faixa etária, a percentagem de indivíduos com CPOD igual ou menor que dois atingiu $87,8 \%$.

Para a amostra como um todo, as freqüências de dentes segundo condição foram as seguintes: hígidos $(71,8 \%)$, cariados $(15,9 \%)$ e
Tabela 1

Distribuição das freqüências absoluta e relativa do índice CPOD em crianças (5 a 12 anos de idade), sexos combinados. Etéñitépa, Mato Grosso, Brasil, 1997.

\begin{tabular}{lccc}
\hline CPOD & $\begin{array}{c}\text { Frequência } \\
\text { absoluta }(\mathrm{n})\end{array}$ & $\begin{array}{c}\text { Frequência } \\
\text { relativa (\%) }\end{array}$ & \% acumulada \\
\hline 0 & 50 & 67,5 & 67,5 \\
1 & 7 & 9,5 & 77,0 \\
2 & 8 & 10,8 & 87,8 \\
3 & 3 & 4,0 & 91,8 \\
4 & 2 & 2,7 & 94,5 \\
5 & 1 & 1,4 & 95,9 \\
7 & 2 & 2,7 & 98,6 \\
8 & 1 & 1,4 & 100,0 \\
Total & 74 & 100,0 & 100,0
\end{tabular}

perdidos $(11,3 \%)$. Chama a atenção a baixa freqüência de dentes obturados $(1,0 \%)$, os quais se concentraram principalmente em homens entre 20-29 anos. Para a dentição decídua, as 109 crianças entre 2 e 13 anos examinadas apresentaram 75,8\% dos dentes hígidos, 22,5\% cariados e 1,7\% com extração indicada. Não foram observados dentes decíduos obturados.

Quanto às condições dos dentes permanentes da arcada superior, os incisivos são os que exibem maior percentagem na condição "cariado" e os molares, na condição "perdido". Os incisivos da arcada inferior apresentam a maior percentagem de hígidos $(94,9 \%)$. Os molares são os dentes em piores condições na arcada inferior.

No que tange aos dentes decíduos da arcada superior, os caninos são os dentes mais preservados, com maior percentagem na condição hígida $(84,3 \%)$. O primeiro molar é o que está em piores condições, com menor percentagem na condição hígido $(66,7 \%)$ e maior, na condição cariado (30,5\%). Na arcada inferior, os incisivos apresentam-se $100 \%$ na condição hígida, seguidos dos caninos (96,4\%). Os molares são os dentes em piores condições na arcada inferior.

Os valores médios de dentes hígidos, do índice CPOD e de seus componentes são mostrados na Tabela 2. A faixa etária 12-14, que inclui a idade de 12 anos, considerada referência pela OMS para efeito de comparações, apresenta CPOD de 3,7. Nas faixas subseqüentes, o CPOD praticamente dobra de uma para outra, alcançando seu valor mais elevado $(19,6)$ aos 50 anos ou mais, revelando a tendência acumulativa da doença cárie. Ao analisar os componentes separadamente, nota-se que o componente obturado $(\mathrm{O})$ é bastante reduzido, o que confirma 
comentários anteriores. Já o componente perdido (P) é relativamente baixo até a faixa etária de 20-29 anos, tornando-se mais proeminente a partir dos 30 anos.

O número médio de dentes decíduos hígidos, cariados (c), perdidos (e), obturados (o) e as médias do índice ceo estão expressos na Tabela 3. O índice ceo mostra-se mais elevado para a idade $6(5,6)$ e 8 anos $(5,3)$. O componente cariado também foi maior nessas idades (5,5 e 4,1 , respectivamente). O componente obturado (o) é igual a zero em todas as idades.

As médias de sextantes afetados de acordo com o ICNTP estão indicadas na Tabela 4. Há o

Tabela 2

Número médio de dentes permanentes hígidos $(H)$, cariados $(C)$, perdidos $(P)$, obturados (O) e índice CPO segundo idade, sexos combinados. Etéñitépa, Mato Grosso, Brasil, 1997.

\begin{tabular}{lrrrrrr}
\hline Idade (anos) & $\mathbf{n}$ & $\mathbf{H}$ & $\mathbf{C}$ & $\mathbf{P}$ & $\mathbf{O}$ & CPO \\
\hline 2 а 5 & 45 & 0,44 & 0,04 & - & - & 0,04 \\
6 а 11 & 52 & 10,67 & 0,84 & - & - & 0,84 \\
12 a 14 & 20 & 23,75 & 3,30 & 0,30 & 0,10 & 3,70 \\
15 а 19 & 25 & 24,80 & 4,20 & 0,40 & - & 4,60 \\
20 a 29 & 39 & 22,92 & 5,28 & 2,28 & 0,97 & 8,53 \\
30 a 39 & 20 & 16,95 & 6,75 & 7,95 & 0,35 & 15,05 \\
40 a 49 & 17 & 17,70 & 6,82 & 7,41 & 0,06 & 14,29 \\
50 ou mais & 10 & 12,40 & 5,90 & 13,70 & - & 19,60
\end{tabular}

Tabela 3

Número médio de dentes decíduos hígidos (h), cariados (c), extraídos/perdidos (e), obturados (o), e do índice ceo segundo idade, sexos combinados. Etéñitépa, Mato Grosso, Brasil, 1997.

\begin{tabular}{rrrcccc}
\hline Idade (anos) & $\mathbf{N}$ & $\mathbf{H}$ & $\mathbf{C}$ & $\mathbf{e}$ & $\mathbf{0}$ & $\mathbf{c e o}$ \\
\hline 2 & 06 & 17,83 & 0,50 & - & - & 0,50 \\
3 & 09 & 17,78 & 2,11 & - & - & 2,11 \\
4 & 16 & 15,19 & 4,56 & 0,19 & - & 4,75 \\
5 & 14 & 14,93 & 4,57 & - & - & 4,57 \\
6 & 13 & 12,46 & 5,54 & 0,08 & - & 5,62 \\
7 & 10 & 10,90 & 3,70 & 0,10 & - & 3,80 \\
8 & 08 & 7,00 & 4,13 & 1,12 & - & 5,25 \\
9 & 07 & 7,00 & 2,29 & 0,14 & - & 2,43 \\
10 & 10 & 3,50 & 2,00 & 0,60 & - & 2,60 \\
11 & 04 & 2,25 & 0,50 & 1,00 & - & 1,50 \\
12 & 08 & 0,12 & - & 0,12 & - & 0,12 \\
13 & 04 & 0,50 & - & - & - & - \\
\hline
\end{tabular}

predomínio de sextantes saudáveis ou com sangramento. Ao examinar as freqüências dos componentes do ICNTP, vê-se que, em geral, a distribuição está concentrada nos componentes hígidos (0), sangramento (1) e tártaro (2), não existindo praticamente casos de indivíduos em estágios avançados da doença periodontal.

A comparação entre os sexos aponta diferenças: enquanto as mulheres apresentam maior percentagem de sextantes com sangramento, há mais homens com tártaro. Na arcada superior, o tártaro concentra-se basicamente nos sextantes posteriores; na inferior, a concentração é no sextante anterior.

Quanto às freqüências dos componentes do ICNTP por sextante, idade e arcada, observa-se que os componentes seguem basicamente a mesma distribuição, ou seja, a concentração dos componentes hígido (0) e sangramento (1) na faixa etária mais jovem e do componente tártaro (2) nos indivíduos mais velhos. Neste componente (tártaro), observa-se diferença importante para a arcada superior nas percentagens entre os sexos na faixa etária mais elevada. Os homens apresentam mais que o dobro de dentes com tártaro nos três sextantes. As bolsas periodontais estão também concentradas na faixa etária mais elevada, apesar das percentagens deste componente serem baixas em relação aos outros do índice. Para a arcada inferior, nota-se que o componente tártaro apresenta elevada concentração nos sextante anteriores tanto na faixa etária jovem como na mais elevada.

Os resultados da classificação de Angle estão indicados na Tabela 5. A análise é dificultada pela grande perda dos primeiros molares, utilizados para estabelecer o tipo de relação entre as arcadas. Em expressiva percentagem de indivíduos não foi possível estabelecer o tipo de relação ( $32 \%$ para os homens e $45 \%$ para as mulheres). De forma geral, ambos os sexos seguem o mesmo padrão de distribuição. Não levando em consideração os casos sem relação de molares, observa-se que a maioria apresenta relação do tipo classe I $(84,3 \%)$, uma pequena parte, classe III $(12,9 \%)$ e uma minoria, classe II (2,8\%). Na faixa etária 12-19 anos, na qual o número de arcadas sem relação de molares é pequeno $(4,4 \%)$, o padrão de distribuição é semelhante. Nas faixas etárias subseqüentes, há grande quantidade de indivíduos sem relação de molares, dificultando afirmações quanto à distribuição dos tipos de relação.

Os dados relativos à má-oclusão estão também expostos na Tabela 5 . A condição “ 0 ”, que indica ausência de má oclusão, foi observada 
na maior parte da amostra $(84,6 \%)$. Apenas $1,3 \%$ da amostra apresentou a condição 2 , que revela problema moderado ou severo de oclusão. Não foram evidenciadas diferenças nas freqüências segundo sexo ou idade. As faixas etárias a partir de 30 anos não apresentam casos de má-oclusão moderada ou severa. Devese considerar, contudo, que ocorrem perdas dentárias com maior freqüência a partir desta idade, influenciando o diagnóstico de oclusão.

Em relação à prótese, a maior parte da amostra não a necessita $(80,7 \%$ na arcada superior e $82,5 \%$ na arcada inferior). A maior necessidade é de próteses parciais $(15,8 \%$ para a arcada superior e $14,9 \%$ para a arcada inferior). A comparação entre os sexos mostra que as mulheres têm necessidade maior de prótese parcial que os homens (respectivamente, $22,5 \%$ e $8,4 \%$ para a arcada superior; $18,3 \%$ e $11,1 \%$ para a arcada inferior).

Quanto à necessidade de tratamento dos dentes permanentes, pode-se dizer que, para a arcada superior, está dividida sobretudo entre os incisivos e os molares. $\mathrm{Na}$ arcada inferior, são os molares que concentram as maiores necessidades de tratamento. Para os dentes decíduos, observa-se que na arcada superior as necessidades estão mais homogeneamente distribuídas entre os grupos dentários - em particular, entre os incisivos e os primeiros e segundos molares. Já na arcada inferior, as necessidades concentram-se quase totalmente nos molares.

\section{Discussão}

Do ponto de vista epidemiológico, a cárie é possivelmente a doença bucal de maior relevância. Sua estreita associação com dieta e estilo de vida também lhe confere relevância antropológica. Estratégias de subsistência e de produção de alimentos empregadas em épocas diversas por diferentes grupos humanos fazem-se refletir na ocorrência e na distribuição das lesões cariosas, em razão de estarem associadas a padrões particulares de dieta e usos diferenciados do aparelho mastigatório.

Há ampla literatura em antropologia biológica e paleopatologia, fundamentada usualmente na análise de remanescentes esqueletais, que aponta para as inter-relações entre transformações nas estratégias de subsistência e na dieta ao longo da história humana e as alterações nas condições de saúde bucal (e presença de cárie, em particular) (Cohen, 1989; Hillson, 1996; Scott \& Turner, 1988). Nesse âmbito, uma temática intensamente investigada diz respeito aos efeitos da transição de uma economia baseada na caça e coleta à agricultura (Cohen \& Armelagos, 1984). Inúmeros estudos de caso demonstram que, em grupos caçadores-coletores, a freqüência da doença é mínima. Já nas economias mistas e, sobretudo, nas baseadas em agricultura, a ocorrência de lesões cariosas é mais expressiva (Cohen, 1989; Cohen \& Armelagos, 1984; Hillson, 1996; Scott \& Turner, 1988). Nessas investigações, o aumento na prevalência de cárie é atribuído usualmente à adoção de dieta mais rica em carboidratos oriundos da agricultura. Além disso, mudanças na forma de preparo dos alimentos, que passaram a ser consumidos preferencialmente cozidos, tornando-os mais moles e adesivos, teriam facilitado o acúmulo de placa, o que eleva o potencial cariogênico.

Tabela 4

Número médio de sextantes afetados (por indivíduo) segundo condições do Índice Periodontal Comunitário (ICNTP), sexos combinados. Etéñitépa, Mato Grosso, Brasil, 1997.

\begin{tabular}{lc}
\hline Código - condição & Sextantes afetados \\
\hline 0 (saudável) & 3,0 \\
$1+2+3+4$ (sangramento) & 1,75 \\
$2+3+4$ (tártaro) & 0,83 \\
$3+4$ (bolsa de $4-5 \mathrm{~mm}$ ) & 0,08 \\
4 (bolsa > ou = 6 mm) & 0,01 \\
9 (sextante excluído) & 0,33 \\
\hline
\end{tabular}

Tabela 5

Distribuição das freqüências absoluta e relativa da classificação de Angle e das condições de má-oclusão segundo sexo. Etéñitépa, Mato Grosso, Brasil, 1997.

\begin{tabular}{lrrr} 
& \multicolumn{2}{c}{ Sexo } & \multicolumn{1}{c}{ Total } \\
& Masc. & \multicolumn{1}{c}{ Fem. } & \\
\hline $\begin{array}{l}\text { Classificação de Angle } \\
\text { Classe I }\end{array}$ & $59(54,6 \%)$ & $59(49,2 \%)$ & $118(51,7 \%)$ \\
Classe II & $3(2,8 \%)$ & $1(0,8 \%)$ & $4(1,8 \%)$ \\
Classe III & $12(11,1 \%)$ & $06(5,0 \%)$ & $18(7,9 \%)$ \\
Sem relação & $34(31,5 \%)$ & $54(45,0 \%)$ & $88(38,6 \%)$ \\
Total & $108(100,0 \%)$ & $120(100,0 \%)$ & $228(100,0 \%)$ \\
Má-oclusão & & & $193(84,6 \%)$ \\
(nenhuma) & & $104(86,7 \%)$ & $32(14,1 \%)$ \\
1 (leve) & $17(15,7 \%)$ & $15(12,5 \%)$ & $3(1,3 \%)$ \\
2 (moderada ou severa) & $2(1,9 \%)$ & $1(0,8 \%)$ & $228(100,0 \%)$ \\
Total & $108(100,0 \%)$ & $120(100,0 \%)$ & \\
\hline
\end{tabular}


Ainda que em escala temporal distinta e envolvendo outros fatores humanos, sociais, econômicos e ambientais, os grupos indígenas contemporâneos, uma vez em contato com sociedades nacionais, também experimentam alterações sócio-econômicas e ecológicas com forte potencial de alterar as condições de saúde bucal (Santos \& Coimbra Jr., 1994; Wirsing, 1988). Diversos estudos realizados no Brasil e em outros países documentam a deterioração das condições de saúde bucal de povos indígenas (Donnelly, 1977; Grim et al., 1994; Tsubouchi et al., 1995). O aumento do consumo de alimentos industrializados - em especial, açúcar e outros tipos de carboidratos fermentáveis - é tido como o principal fator envolvido na alteração do perfil de saúde bucal dessas populações. Há ampla literatura que indica a estreita e positiva relação entre consumo de açúcar e prevalência de cárie (Gustafsson et al., 1954; Newbrum, 1982; Pilot, 1988; Sheihan, 1984; Woodward \& Walker, 1994).

O caso dos Xavánte de Etéñitépa é representativo desta tendência. Segundo Freitas \& Oliveira (1955) e Neel et al. (1964), a dieta Xavánte era propiciadora de boas condições dentárias. Constituída de muitos alimentos crus, exigia o uso intensivo dos dentes, o que agia como fator inibidor do desenvolvimento da cárie. Segundo Armelagos (1969), a abrasão provocada pela mastigação de alimentos duros promove a diminuição dos microorganismos da superfície dental, prevenindo o estágio inicial da doença; a atrição dos dentes elimina sulcos e fissuras, dificultando a retenção de placa.

As alterações na dieta dos Xavánte intensificaram-se a partir dos anos 70, quando houve a implantação de um "projeto de desenvolvimento comunitário" (conhecido como "Projeto Xavánte”), no qual um dos objetivos era a implementação de agricultura comercial nas áreas indígenas. Visava tornar os Xavánte auto-suficientes economicamente mediante a produção de excedentes comercializáveis (Santos et al., 1997; Silva, 1992). Essa iniciativa, que não atingiu suas metas principais e foi posteriormente abandonada, introduziu definitivamente o arroz na dieta Xavánte, que constitui atualmente a base de sua alimentação. Segundo Sreenbny (1983), há evidências de que o potencial cariogênico do milho - alimento importante no passado para a dieta dos Xavánte - seja menos pronunciado que o do arroz.

Paralelamente, ao longo das duas últimas décadas, houve incremento significativo da presença de alimentos industrializados - em particular, açúcar, óleo de cozinha e sal -, como também de refrigerantes, doces, biscoitos, entre outros, na dieta Xavánte (Flowers, 1983; Gugelmin, 1995; Santos et al., 1997). Segundo Santos et al. (1997), em termos percentuais, a presença de itens alimentares industrializados na dieta dos Xavánte de Etéñitépa cresceu de $2 \%$ para $9 \%$ entre 1976/1977 e 1994. Esses autores indicam que o incremento no consumo está associado à presença de novas fontes de renda - salários e pensões - e à maior facilidade de acesso aos núcleos urbanos regionais.

Estudos anteriores realizados entre os Xavánte de Pimentel Barbosa em 1962 e 1991, coletaram dados referentes à saúde bucal (Neel et al., 1964; Pose, 1993). Ainda que seja preciso ter cautela ao comparar os resultados desses autores com aqueles do presente inquérito em virtude de eventuais diferenças metodológicas, estes constituem as únicas fontes disponíveis que permitem verificar a ocorrência de tendências ao longo do tempo. Neel et al. (1964) observaram freqüência mínima de cárie entre os Xavánte. Na ocasião, relataram que mantinham dieta baseada na caça e coleta de frutos e raízes silvestres, complementados pela agricultura baseada no milho, feijão e abóbora. Segundo Neel et al. (1964), os níveis de cárie observados eram próximos aos reportados para aborígines australianos que ainda não tinham sido expostos à dieta européia. A Tabela 6 mostra que os valores médios do CPOD são consistentemente mais elevados para 1997 se comparados aos reportados em levantamentos anteriores. Os resultados dos dois levantamentos conduzidos nos anos 90 também apontam para tendência de aumento dos valores do índice ceo (Tabela 7). Portanto, há evidências de que a tendência foi de deterioração das condições de saúde bucal dos Xavánte de Etéñitépa ao longo do tempo, algo esperado à luz da história recente do grupo.

A idade de 12 anos, considerada referência pela OMS para comparações da prevalência de cárie, apresentou CPOD de 2,6 em 1997. Este valor está dentro da faixa tida como de prevalência baixa (CPOD entre 1,2 a 2,6) (vide Pinto, 1992). Em relação a parâmetros nacionais, a cifra observada em Etéñitépa é menor do que a estimada para o Brasil $(3,1)$ e para a região Centro-Oeste $(2,8)$, as quais derivam de levantamentos realizados nas capitais (MS, 1996). É preciso considerar esses dados com cautela quanto à representatividade para o país, uma vez que não incluem as zonas rurais, onde os valores de CPOD devem ser mais elevados.

Se, por um lado, os valores do CPOD para os Xavánte ainda se encontram em níveis considerados baixos, os resultados deste estudo apontam claramente para um quadro de dete- 
Médias e desvios-padrão (DP) do índice CPOD resultantes de levantamentos epidemiológicos realizados entre os Xavánte em diferentes ocasiões, segundo idade, sexos combinados. Etéñitépa, Mato Grosso, Brasil.

\begin{tabular}{|c|c|c|c|c|c|c|c|c|}
\hline \multirow[t]{2}{*}{ Idade (anos) } & \multicolumn{2}{|c|}{$1962^{\star}$} & \multicolumn{3}{|c|}{$1991^{\star \star}$} & \multicolumn{3}{|c|}{1997} \\
\hline & $\mathrm{n}$ & média & $\mathrm{n}$ & média & $\mathrm{DP}$ & $n$ & média & DP \\
\hline 6 a 12 & 14 & 0,21 & 35 & 0,37 & 0,77 & 60 & 1,08 & 1,89 \\
\hline 13 a 19 & 21 & 0,30 & 25 & 1,16 & 1,60 & 37 & 4,54 & 3,79 \\
\hline 20 a 34 & 17 & 0,71 & 23 & 8,13 & 6,00 & 46 & 9,72 & 7,17 \\
\hline 35 a 44 & 05 & 2,40 & 10 & 9,10 & 5,70 & 24 & 14,25 & 6,09 \\
\hline 45 ou mais & 03 & 3,60 & 18 & 13,78 & 7,68 & 16 & 17,75 & 6,32 \\
\hline
\end{tabular}

* O cálculo do CPO-D, a partir do levantamento conduzido em 1962 por Neel et al. (1964),

foi realizado por Pose (1993: 46).

** Dados de 1991 obtidos de Pose (1993: 110).

rioração das condições de saúde bucal ao longo do tempo - principalmente, de cárie - em praticamente todas as faixas etárias. Trata-se de tendência inversa ao que se tem observado em estudos epidemiológicos conduzidos em várias regiões do Brasil, nas quais os índices de cárie vêm apresentando tendência de queda ao longo do tempo (Dini et al., 1999; Freysleben et al., 2000; MS, 1996; Narvai et al., 2000).

A pesquisa em Etéñitépa revelou freqüência baixa de dentes obturados, achado que era esperado, uma vez que os Xavánte não têm praticamente acesso a serviços odontológicos. Há de se ressaltar que os poucos indivíduos com dentes obturados são principalmente homens na faixa etária de 20-29 anos, o que aponta para oportunidades diferenciadas de acesso a tratamentos curativos segundo idade e sexo.

Em relação às doenças periodontais, é interessante observar que, apesar de não haver uma forma de higienização oral sistemática e difundida entre os Xavánte, o ICNTP sugere baixa ocorrência de doença periodontal em estágios avançados. Esta situação também foi observada por Niswander (1967) para os Xavánte de Simões Lopes. Em Etéñitépa, a gengivite é mais freqüente nas mulheres; entre os homens, sobressai o tártaro. Contudo, os pequenos tamanhos de amostra não permitem conclusões definitivas quanto a diferenças entre os sexos para esses parâmetros. É pouco expressiva a ocorrência de bolsas periodontais e de dentes com mobilidade em ambos os sexos, mesmo entre os idosos. Tal perfil pode ser reflexo de resistência maior a fatores irritativos locais (placa, tártaro) e/ou advir de aspectos não investigados neste estudo, como flora bucal ou possíveis efeitos benéficos da estimulação dos tecidos de suporte, promovidos por dieta ainda em parte constituída de alimentos
Tabela 7

Médias e desvios-padrão (DP) do índice ceo resultantes de levantamentos epidemiológicos realizados entre os Xavánte em diferentes ocasiões, segundo idade, sexos combinados. Etéñitépa, Mato Grosso, Brasil.

\begin{tabular}{ccccccc}
\hline Idade (anos) & & $\begin{array}{c}1991^{*} \\
\text { média }\end{array}$ & DP & $n$ & $\begin{array}{c}1997 \\
\text { média }\end{array}$ & DP \\
\hline 2 & $n$ & - & - & 06 & 0,50 & 0,84 \\
3 & - & - & - & 09 & 2,11 & 4,51 \\
4 & - & - & - & 16 & 4,75 & 3,23 \\
5 & - & - & - & 14 & 4,57 & 3,69 \\
6 & 05 & 4,00 & 3,54 & 13 & 5,61 & 4,15 \\
7 & 03 & 1,00 & 1,00 & 10 & 3,80 & 2,15 \\
8 & 08 & 1,87 & 2,48 & 08 & 5,25 & 3,02 \\
9 & 03 & 3,00 & 5,20 & 07 & 2,43 & 1,81 \\
10 & 06 & 1,50 & 0,84 & 10 & 2,60 & 2,01 \\
11 & - & - & - & 04 & 1,50 & 1,91 \\
12 & - & - & - & 08 & 0,12 & 0,35 \\
\hline
\end{tabular}

* Dados de 1991 obtidos de Pose (1993:111).

duros e fibrosos. Essas observações a partir dos dados coletados em Etéñitépa estão de acordo com estudos recentes a respeito de periodontopatias, segundo os quais a gengivite e o tártaro não provocariam necessariamente a periodontite, podendo haver casos prolongados de gengivite sem evolução da doença para os demais tecidos de suporte dentário (Pilot, 1988; Renson,1989; WHO, 1992).

No que se refere aos padrões de oclusão, foi observado que, em sua quase totalidade, os Xavánte de Etéñitépa possuem arcadas bem formadas e desenvolvidas de forma harmoniosa. A maioria dos indivíduos (84,6\%) não apresenta oclusopatias. Em uma parcela da população observou-se leve má oclusão, constituída ge- 
ralmente por pequeno apinhamento dentário anterior ou giroversão $(14,1 \%)$. As arcadas são geralmente ocluídas em classe I de Angle $(84,3 \%)$, uma parte mínima em classe II $(2,8 \%)$, e pequena parcela em classe III $(12,9 \%)$. Nos anos 60, 95\% da população Xavánte de Simões Lopes apresentava oclusão ideal, com arcadas ocluídas em classe I de Angle, dentes em bom alinhamento, overjet (distância entre os incisivos superiores e inferiores em oclusão) menor que 4-5 mm e overbite (distância entre as bordas incisais dos incisivos superiores e inferiores em oclusão) menor que $2 / 3$ dos incisivos inferiores (Niswander, 1967). Este autor não observou casos de má-oclusão classe II ou III de Angle. Pose (1993) observou elevada percentagem de pessoas com oclusão normal em Etéñitépa, ainda que a freqüência de casos classe I de Angle (91,9\%) fosse pouco inferior à observada por Niswander, com conseqüente aumento nas classes II $(2,3 \%)$ e III $(5,8 \%)$.

Tomados em conjunto, os dados mencionados acima podem ser interpretados como sugerindo tendência de aumento nos níveis de oclusopatias entre os Xavánte. Deve-se salientar, contudo, que não é possível estabelecer com segurança os fatores relacionados às mudanças nos perfis de oclusão com base nas evidências disponíveis. Uma linha explicativa para o aumento de oclusopatias que se observa em populações humanas de diversas partes do mundo advoga a influência de alterações nos padrões de dieta e estilo de vida ao longo do tempo - incluindo a tendência de diminuição no consumo de alimentos mais duros e fibrosos - e seus impactos negativos sobre as estruturas mastigatórias (Corruccini, 1984).
Grupos humanos cuja economia baseia-se em atividades de caça, coleta e agricultura, tendem a apresentar menores níveis de oclusopatias do que aqueles que vivem em ambientes urbanos e industrializados (Corruccini, 1984). Esse modelo que enfatiza a dimensão ambiental e seu sinergismo com o desenvolvimento do complexo orofacial, é plausível para explicar o incremento nos níveis de oclusopatias entre os Xavánte. Contudo, é preciso interpretar os dados com bastante cautela, uma vez que os Xavánte de Etéñitépa vêm apresentando importante aumento nos níveis de perdas dentárias, o que, por si, pode influenciar as freqüências de oclusopatias (Frazão, 1999). Pesquisas mais detalhadas serão necessárias para chegar a conclusões melhor embasadas quanto às causas relacionadas à aparente elevação nos níveis de oclusopatias entre os Xavánte.

Em conclusão, a saúde bucal dos Xavánte de Etéñitépa tem experimentado alterações, em especial quanto à prevalência de cárie nas últimas décadas. Nesse período houve expressivo aumento no consumo de alimentos industrializados, incluindo açúcar refinado e outros produtos de alto potencial cariogênico. É importante salientar que a dieta dos Xavánte de Etéñitépa ainda mantém muitas características tradicionais, na qual há presença de raízes, frutos, peixes e caça, favorecendo uma situação de saúde bucal ainda razoável, com prevalência de cárie considerada moderada. Diante do quadro de deterioração das condições de saúde bucal, é de fundamental importância que sejam implementados programas preventivos, entre outras iniciativas, visando a difundir a prática da higienização sistemática e o uso de dentifrícios fluoretados.

\section{Agradecimentos}

Nossos agradecimentos aos Xavánte, pelo interesse em participar do estudo. Esta pesquisa foi realizada enquanto o autor principal (Arantes) era bolsista do CNPq no Programa de Mestrado em Saúde Pública da Escola Nacional de Saúde Pública, Fundação Oswaldo Cruz. O trabalho foi parcialmente financiado pelo Programa de Apoio à Pesquisa Estratégica em Saúde (PAPES), da Fundação Oswaldo Cruz. Agradecemos também o apoio da Kolynos do Brasil pelo fornecimento de material para o atendimento clínico e de higienização bucal. 


\section{Referências}

ARMELAGOS, G. J., 1969. Disease in ancient Nubia. Science, 163:255-259.

ASHTON-TATE, 1985. Dbase III Plus Version 1.0 IBM/ MSDOS. Washington, DC: Ashton-Tate.

AYRES, M. \& SALZANO, F. M., 1972. Health of Brazilian Cayapo indians. Tropical and Geographical Medicine, 24:178-185.

COHEN, M. N., 1989. Health and the Rise of Civilization. New Haven: Yale University Press.

COHEN, M. N. \& ARMELAGOS, G. J. (eds.)., 1984. Paleopathology at the Origins of Agriculture. New York: Academic Press.

COIMBRA Jr., C. E. A. \& SANTOS, R. V., 2000. Saúde, minorias e desigualdade: Algumas teias de interrelações, com ênfase nos povos indígenas no Brasil. Ciência \& Saúde Coletiva, 5:125-132.

CORRUCCINI, R. S., 1984. An epidemiologic transition in dental occlusion in world populations. American Journal of Orthodontics, 86:419-426.

DETOGNI, A., 1994. De volta às origens. Revista da Associação Brasileira de Odontologia, 2:138-148.

DINI, E. L; FOSCHINI, A. L. R.; BRANDÃO, I. M. G. \& SILVA, S. R. C., 1999. Changes in caries prevalence in 7-12-year-old children from Araraquara, São Paulo, Brazil: 1989-1995. Cadernos de Saúde Pública, 15:617-621.

DONNELLY, C. J., 1977. Plaque, caries, periodontal diseases, and acculturation among Yanomámi Indians, Venezuela. Community Dentistry and Oral Epidemiology, 5:30-39.

FLOWERS, N. M., 1983. Seasonal factors in subsistence, nutrition, and child growth in a central Brazilian Indian community. In: Adaptive Responses of Native Amazonians (R. B. Hames \& W. H. Vickers, eds.), pp. 357-390, New York: Academic Press.

FRAZÃO, P., 1999. Epidemiologia da Oclusão Dentária na Infância e os Sistemas de Saúde. Tese de Doutorado, São Paulo: Faculdade de Saúde Pública, Universidade de São Paulo.

FREITAS, S. A. F. \& OLIVEIRA, N. B., 1955. Estudo sobre o estado nutritivo dos Xavánte. Revista Brasileira de Medicina, 12:565-567.

FREYSLEBEN, G. R.; PERES, M. A. A. \& MARCENES, W., 2000. Prevalência de cárie e CPO-D médio em escolares de doze a treze anos de idade nos anos de 1971 e 1997, região Sul, Brasil. Revista de Saúde Pública, 34:304-308.

GIACCARIA, B. \& HEIDE, A., 1972. Xavánte: Povo Autêntico. São Paulo: Editora Salesiana Dom Bosco.

GRIM, C. W.; BRODERICK, E. B.; JASPER, B. \& PHIPPS, K. R., 1994. A comparison of dental caries experience in Native American and Caucasian children in Oklahoma. Journal of Public Health Dentistry, 54:220-227.

GUGELMIN, S. A., 1995. Nutrição e Alocação de Tempo dos Xavánte de Pimentel Barbosa, Mato Grosso: Um Estudo em Ecologia Humana e Mudanças. Dissertação de Mestrado, Rio de Janeiro: Escola Nacional de Saúde Pública, Fundação Oswaldo Cruz.

GUSTAFSSON, B. E.; QUENSEL, C. E.; LANKE, L. S.; LUNDQVIST, C.; GRAHNÉN, H.; BONOW, B. E. \& KRASSE, B., 1954. The Vipeholm dental caries study. The effect of different levels of carbohidrate intake on caries activity in 436 individuals observed for five years. Acta Odontologica Scandinavica, 11:232-364.

HILLSON, S., 1996. Dental Anthropology. Cambridge: Cambridge University Press.

HIRATA, J.; BERGAMASCHI, O.; OLIVEIRA, A.; LAZARO, A.; MARTINS, C.; BOSCO, L. \& ANDO, T., 1977. Estudo de prevalência de cárie em crianças indígenas do Parque Nacional do Xingú. Revista da Faculdade de Odontologia de São Paulo, 15:189198.

MAYBURY-LEWIS, D., 1967. Akwe-Shavante Society. Oxford: Clarendon Press.

MS (Ministério da Saúde), 1988. Levantamento Epidemiológico em Saúde Bucal: Brasil, Zona Urbana. Brasília: Secretaria Nacional de Programas Especiais de Saúde. Divisão Nacional de Saúde Bucal, Ministério da Saúde.

MS (Ministério da Saúde), 1996. Levantamento Epidemiológico em Saúde Bucal. Brasília: Secretaria Nacional de Programas Especiais de Saúde, Divisão Nacional de Saúde Bucal, Ministério da Saúde.

NARVAI, P. C.; CASTELLANOS, R. \& FRAZÃO, P., 2000. Prevalência de cárie em dentes permanentes de escolares do Município de São Paulo, SP, 19701996. Revista de Saúde Pública, 34:196-200.

NEEL, J. V.; SALZANO, F. M.; JUNQUEIRA, P. C.; KEITER, F. \& MAYBURY-LEWIS, D., 1964. Studies on the Xavánte Indians of the Brazilian Mato Grosso. American Journal of Human Genetics, 16:52-140.

NEWBRUM, E., 1982. Sugar and dental caries: A review of human studies. Science, 217:418-423.

NISWANDER, J. D., 1967. Further studies on Xavánte Indians. VII. The oral status of the Xavántes of Simões Lopes. American Journal of Human Genetics, 19:543-553.

NORMANDO, A. D. C. \& ARAÚJO, I. C., 1990. Prevalência de cárie dental em uma população de escolares da região amazônica. Revista de Saúde Pública, 24:294-299.

PEREIRA, C. \& EVANS, H., 1975. Occlusion and attrition of the primitive Yanomámi Indians of Brazil. Dental Clinics of North America, 19:485-498.

PILOT, T., 1988. Trends in oral health: A global perspective. New Zealand Dental Journal, 84:40-45.

PINTO, V. G., 1990. Saúde Bucal. Panorama Internacional. Brasília: Secretaria Nacional de Programas Especiais de Saúde, Ministério da Saúde.

PINTO, V. G., 1992. Saúde Bucal. Odontologia Social e Preventiva. São Paulo: Livraria Santos Editora.

POSE, S. B., 1993. Avaliação das Condições de Saúde Oral dos Índios Xavánte, Brasil Central. Dissertação de Mestrado, Rio de Janeiro: Escola Nacional de Saúde Pública, Fundação Oswaldo Cruz.

RENSON, C. E., 1989. Global changes in caries prevalence and dental manpower requirements:3. The effects on manpower needs. Dental Update, 16: 382-389.

SANTOS R. V. \& COIMBRA Jr., C. E. A., 1994. Saúde e Povos Indígenas. Rio de Janeiro: Editora Fiocruz.

SANTOS, R. V.; FLOWERS, N.; COIMBRA Jr., C. E. A. \& GUGELMIN, S. A., 1997. Tapirs, tractors, and tapes: The changing economy and ecology of the 
Xavánte Indians of Central Brazil. Human Ecology, 25:545-566.

SCOTT, G. R. \& TURNER, C. G., 1988. Dental anthropology. Annual Review of Anthropology, 17:99126.

SHEIHAM, A., 1984. Changing trends in dental caries. International Journal Epidemiology, 13:142-147.

SILVA, A. L., 1992. Dois séculos e meio de história Xavánte. In: História dos Índios no Brasil (M. C. Cunha, org.), pp. 357-378. São Paulo: Companhia das Letras.

SREEBNY, L. M., 1983. Cereal availability and dental caries. Community Dentistry and Oral Epidemiology, 11:148-155.

TRICERRI, F. J., 1985. Breve passagem odontológica entre índios do Alto Solimões, Amazônia, Brasil. Revista da Fundação SESP, 30:151-160.

TSUBOUCHI, J.; TSUBOUCHI, M.; MAYNARD, R. J.; DOMOTO, P. K \& WEINSTEIN, P., 1995. A study of dental caries and risk factors among Native American infants. Journal of Dentistry for Children, 62:283-287.

TUMANG, J. A. \& PIEDADE, E. F., 1968. Carie dental, doenças periodontais e higiene oral em indígenas brasileiros. Boletim de la Oficina Sanitária Panamericana, 64:103-109.
VIEGAS, Y. \& VIEGAS, A. R., 1988. Prevalência de cárie dental em Barretos, SP, Brasil, após dezesseis anos de fluoretação da água de abastecimento público. Revista de Saúde Pública, 22:25-35.

WALKER, A. R. P., 1987. Changes in caries epidemiology and other diseases. British Dental Journal, 162:452-453.

WHO (World Health Organization), 1972. Etiología y Prevención de la Caries Dental. Serie de Informes Tecnicos, 494. Geneva: WHO.

WHO (World Health Organization), 1987. Oral Health Surveys - Basic Methods. Geneve: WHO.

WHO (World Health Organization), 1992. Avances Recientes en Salud Bucodental. Serie de Informes Técnicos no. 826, Geneva: WHO.

WHO (World Health Organization), 1994. Epi Info 6.02. A Word Processing, Database, and Statistcs System for Epidemiology on Microcomputers. Geneva: WHO.

WINTER, G. B., 1990. Epidemiology of dental caries. Archives of Oral Biology, 35(Sup.): 1S-7S.

WIRSING, R., 1985. The health of traditional societies and effects of acculturation. Current Anthropology, 26:303-322.

WOODWARD, M. \& WALKER, A. R. P., 1994. Sugar consumption and dental caries: Evidence from 90 countries. British Dental Journal, 176:297-302. 